\title{
Optical Transition, Excitation, and Emission Properties of Poly(N-Vinlycarbazole) Blended with Poly(Vinylidene Fluoride-co-Hexafluoropropene) and Polyvinylpyrrolidone
}

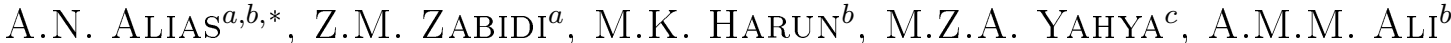 \\ ${ }^{a}$ Faculty of Applied Science, Universiti Teknologi Mara (Perak) Kampus Tapah 35400 Tapah Road, Perak, Malaysia \\ ${ }^{b}$ Ionic Materials \& Devices (iMADE) Research Laboratory, Institute of Sciences, \\ Universiti Teknologi MARA 40450 Shah Alam, Selangor D.E., Malaysia \\ ${ }^{c}$ Faculty of Defense Science \& Technology, Universiti Pertahanan Nasional Malaysia, Kem Sg. Besi, \\ 57000 Kuala Lumpur, Malaysia

\begin{abstract}
We present the results concerning the excitation and emissions spectra of Poly (N-carbazole) (PVK) blended with poly(vinylidene fluoride-co-hexafluoropropene), (PVDF-HfP) and polyvinylpyrrolidone (PVP). Thin films of PVK blended with PVDF-HfP (PVK:PVDF-HfP) and PVP (PVK:PVP) were prepared using doctor blade technique on spectrosil substrate. The influences of polymer blends on the excitation and emission spectra were and emission for each sample. The possible energy transfer and recombination mechanisms have been related with singlet-singlet semi-empirical INDO electronic calculation and FTIR measurements.
\end{abstract} \\ observed under UV excitation source of a xenon lamp. The result shows a discrepancy in the maximum excitation
}

DOI: $10.12693 /$ APhysPolA.127.1430

PACS: 07.60.-j , 31.15.bu , 81.05.Lg, 82.35.Lr

\section{Introduction}

Poly(N-vinlycarbazole) (PVK) is a well-known nonconjugated polymer for polymer light emitting diode (PLED), photoconductive, photorefractive, lithium-ion battery and photovoltaic (PV) applications [1]. In PLED and PV applications, PVK is used as a hole transport layer because of its high hole mobility. All these applications are enabled by the existence of carbazole groups that are useful in opto-electronics and photonics. PVK can be mixed with organic and inorganic salts or compounds or embedded with nanoparticles to achieve new optical properties and optimized output in photonics and opto-electronics [2,3]. Crosslinking of PVK with other conjugated polymers can also realize new optical properties of PVK [4].

Polymer blending is a simple, well-known technique of creating new solid materials or composites with more enhanced properties than those of homopolymers. In semiconductor application, polymer blending is widely used to optimize the performance of opto-electronic devices. The optical properties of polymer can be modulated by adding some dopants to the host polymer or blending with other polymers. Optical characterizations of polymers are vital tools for understanding the electronic properties of polymers [5]. Optical absorption and luminescence spectroscopy rely on electron transition from the

\footnotetext{
* corresponding author; e-mail: ahmadnazib111@ppinang . uitm.edu.my
}

highest occupied molecular orbital to the lowest unoccupied molecular orbital or on the nonlinear optical properties of a polymeric material.

Pertaining to the alteration described above, we have blended PVK with another host polymer which is poly(vinylidene fluoride-co-hexafluoropropene) (PVDFHFP) or polyvinyl pyrrolidone (PVP), due to its own interesting physical properties. We anticipate that by mixing the polymer luminescence (PVK) with another host polymer, it will change electronic properties of the active layer. In this paper, we report the effect of polymer blends on the excitation and emission spectra of PVK:PVDF-HfP and PVK:PVP blending system. By investigating emission and excitation properties of these polymer blends, it is anticipated to provide some vital information for any PLED applications. The possible energy transfer has been related with singlet-singlet semiempirical INDO electronic calculation and FTIR measurements.

\section{Methodology \\ 2.1. Experimental}

PVK, PVDF-HfP and PVP were purchased from Sigma-Aldrich. Two polymer blend systems consisting of PVK blended with PVDF-HfP (PVK:PVDF-HfP) and PVK blended with PVP (PVK:PVP) in the same weight ratio (1:1) were prepared. Pure PVK was also prepared as a control. PVK, PVK:PVDF-HfP and PVK:PVP were dissolved in $10 \mathrm{ml}$ dimethylformamide and stirred for $6 \mathrm{~h}$ at room temperature $\left(30^{\circ} \mathrm{C}\right)$. Each solution was coated onto a spectrosil substrate using doctor blade technique to form a thin film. Prior to coating, the spectrosil substrate was cleaned with Decon 90, rinsed with deionized 
water and then ultrasonicated with acetone. The film was then dried in a vacuum oven at $55{ }^{\circ} \mathrm{C}$ for $2 \mathrm{~h}$ to remove the solvent. The thickness of the thin films was measured by using reflectometry spectroscopy filmetrics F20 Series. The average thickness of PVK, PVK:PVDFHFP and PVK:PVP thin films was 418.5, 421.5 and 420.0 $\mathrm{nm}$ respectively. Optical absorption and transmittance were measured using Varian Cary 5000 uv-vis spectrophotometer between the wavelengths of 200 and $800 \mathrm{~nm}$. The photoluminescence measurement was conducted using Jobin Yvon Horiba Spex Fluorolog-3 with 450 W Xenon lamp as an excitation source. Fourier Transform Infrared (FTIR) spectroscopy was carried out using ATR Perkin Elmer 400 in the wavelength range from 550 to $4000 \mathrm{~nm}$.

\subsection{Electronic calculation}

The monomeric unit of molecular structure for PVK, PVP and PVDF-HfP oligomer was drawn using the "worksheet' Material Studio ${ }^{\circledR}$ interface. When the desired molecular structure was drawn, geometry optimizations were performed using Parametric Method Number 3 (PM3). The electronic structure of the monomeric unit of molecular structure for PVK, PVP and PVDFHfP was calculated using Zerner Modification intermediate neglect of differential overlap (ZINDO) calculation [6]. The empirical parameter INDO/1 were used with implementing single configuration interaction. All calculation was performed with the VAMP Material Studio Program package.

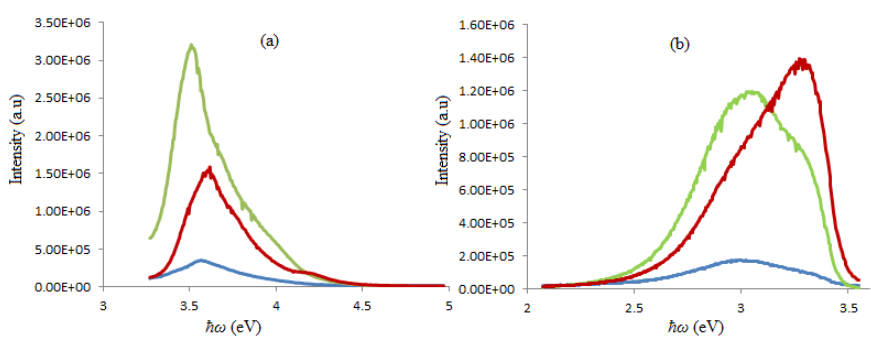

Fig. 1. (a) excitation and (b) emission spectrum for PVK (blue), PVK:PVDF-HfP (red) and PVK:PVP (green).

\section{Results}

\subsection{Emission and excitation spectra}

The luminescence excitation spectrum was measured to characterize the excited electron states of complex molecules which are absorbing the light source near the band gap. The luminescence excitation spectrum also measures the relation between the luminescence emission intensity and the energy of excitation. The maximum excitation energy for PVK, PVK:PVDF-HfP and PVK:PVP (at $415 \mathrm{~nm}$ ) is 3.565, 3.510 and $3.617 \mathrm{eV}$ respectively, as shown in Fig. 1. While the maximum emission energy for PVK, PVDF-HfP and PVK:PVP (at $325 \mathrm{~nm}$ ) is $2.983,3.056$ and $3.274 \mathrm{eV}$ respectively. As revealed in Fig. 1, the maximum excitation spectra are different at the fundamental absorption edge. This is due to the fact, that excitation spectrum strongly depends on surface of recombination, quantum efficiency and absorption depth [7]. The energy difference between maximum excitation and emission energy is known as the Stokes shift. This Stokes shift is related with the coupling energy between electron and phonon $(W)$ by $2 W[8]$. The value of $W$ for PVK, PVK:PVDF-HfP and PVK:PVP is $0.291,0.227$ and $0.1715 \mathrm{eV}$, respectively. PVK has the highest Stokes shift followed by PVK:PVDF-HfP and PVK:PVP. This implies that PVK has a broad emission peak compared to PVK:PVDF-HfP and PVK:PVP.

As depicted in Fig. 1, all polymer systems have different excitation and emission spectra. For excitation spectrum, PVK:PVDF-HfP (Fig. 1a) has the highest excitation intensity, followed by PVK:PVP and PVK. The emission spectrum (Fig. 1b) for each polymer system has a tilted Gaussian shape. It can be observed that PVK: PVP has the highest emission intensity, followed by PVK:PVDF-HfP and PVK. For the PVK:PVDF-HfP, there is a shoulder at a lower wavelength. While for PVK:PVP, the shoulder appears at a higher wavelength. These shoulders are attributed to vibronic transition with different quantized vibronic energy levels [9]. The deviations in emission intensity and in the maximum emission energy are due to the changes of electronic structure in polymer blending system.

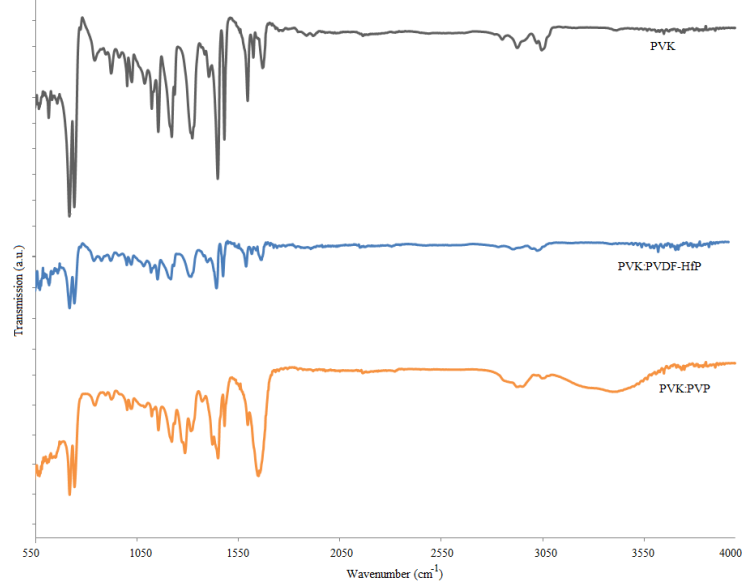

Fig. 2. FTIR spectra of PVK, PVK:PVDF-HfP and PVK:PVP

\subsection{FTIR spectra}

FTIR, or vibration spectroscopy, provides reliable evidence for the occurrence of complexation in thin films, i.e., confirmation of complexation between polymer blends. Figure 2 shows the FTIR spectra of PVK, PVK:PVDF-HfP and PVK:PVP. The main vibration absorption peaks of PVK at 3046, 2926, 1624, 1596.5, 1449, $1405,1323,1222,1123.5-1156,742.5$, and $717.5 \mathrm{~cm}^{-1}$ are assigned as follows: aromatic $\mathrm{C}-\mathrm{H}$ stretching, aliphatic $\mathrm{C}-\mathrm{H}$ stretching, $\mathrm{C}-\mathrm{C}$ stretching in benzene ring, $\mathrm{C}-\mathrm{C}=\mathrm{C}$ stretching in benzene ring, framework vibrational of aromatic ring, $\mathrm{CH}_{2}$ deformation of vinyl group, $\mathrm{CH}$ plane 
deformation of vinyl group, $\mathrm{CN}$ vibrational framework stretching of aromatic ring, $\mathrm{CH}$ in plane deformation of aromatic ring, as well as $\mathrm{CH}_{2}$ rocking vibrational, caused by tail-to-tail addition and ring deformation of substituted aromatic structural [10, 11]. Blended PVK with PVDF-HfP or PVP induces significant changes in the FTIR spectra, i.e., decrease of peak intensity of vibration absorption as well as accretions and reductions in vibration frequencies, compared with the pure PVK. According to literature [12], the main absorption peaks of PVDF-HfP are positioned at 1797, 1417, 1290-1060, 882 , and $839 \mathrm{~cm}^{-1}$, and can be assigned to $-\mathrm{CF}_{2}=\mathrm{CF}_{2}-$ group, $-\mathrm{CH}_{3}$ asymmetric bending, $\mathrm{CF}$ and $\mathrm{C}-\mathrm{F}_{2}$ stretching vibrations, vinylidene group of the polymer, and $\mathrm{C}-$ $\mathrm{F}_{2}$ asymmetric stretching vibration. Most peak intensities of the main carbazole vibration absorption for pure PVK decrease after blending with PVDF-HfP, as shown in Fig. 2. The PVDF-HfP sample also shows a lower vibration absorption peak, compared to the PVK and PVK:PVP. The vibration frequency at $1221-1155 \mathrm{~cm}^{-1}$ also increases, which can be attributed to $\mathrm{CF}$ and $\mathrm{C}-$ $\mathrm{F}_{2}$ stretching vibration and $\mathrm{C}-\mathrm{F}_{2}$ group. A peak is also observed at $838 \mathrm{~cm}^{-1}$, which is assigned to asymmetric stretching vibration. The vibration frequencies at 3046 and $2926 \mathrm{~cm}^{-1}$, assigned to aromatic and aliphatic $\mathrm{C}-\mathrm{H}$ stretching, are diminished in PVK:PVDF-HfP. For PVK:PVP, most of the main carbazole vibration absorption frequencies appear in the FTIR spectra. However, the vibration absorption intensity of PVK:PVP is lower than that of PVK. Accession in the vibration absorption frequency is observed, and broad bands at 3408, 1645, 1156 , and $1021 \mathrm{~cm}^{-1}$ can be assigned as follows: $\mathrm{OH}$ stretching, stretching vibration of $\mathrm{C}=\mathrm{O}, \mathrm{C}-\mathrm{O}$ stretching, and $\mathrm{C}-\mathrm{N}$ stretching, respectively [13].

\section{Discussion}

Figure 1 shows some changes in the excitation and emission spectra of the polymer blend systems probably caused by the following few mechanisms. Initially, energy is transferred from PVP and PVDF-HfP to PVK. According to the Forster theory, energy transfer can be achieved if the excitation spectrum of PVK overlaps with the emission spectrum of PVP/PVDF-HfP [14]. To investigate the energy transfer of polymer-polymer blends, we use molecular quantum chemical calculation using ZINDO method. In this calculation, an assumption that the basic unit of molecular monomer is involved in photophysical process was taken. According to Forster model, singlet-singlet energy transfer could occur due to the interaction of dipole-dipole moment resonance between donor and acceptor [15]. From the quantum chemical calculation for each PVP and PVDF$\mathrm{HfP}$, the lowest singlet energy level $\left(\mathrm{S}_{1}\right)$ is higher compared with that of PVK (Fig. 3). Thus, it can be said that PVP and PVDF-HfP has become the sensitizer (donor), while PVK has become the acceptor. After the occurrence of excitation process, the sensitizer in excited states will eventually convert to the lowest singlet excited state. When the energetic coupling between PVP/PVDF-HfP and PVK is sufficient, the energy transfer will be executed using resonance inductive coupling. It is also found that PVK:PVDF-HfP has a higher energy differences of singlet-singlet transition compared with PVK:PVP. Thus, this makes PVK:PVDF-HfP to require more energy to excite donor from ground state to the excited state. For emission spectrum, experimental result shows that PVK:PVP has the highest emission intensity. Plausible explanations are due to the intra- and inter-molecular interaction for multichromphoric system and spin orbital coupling, which were not taken into account in our molecular orbital calculation [16].

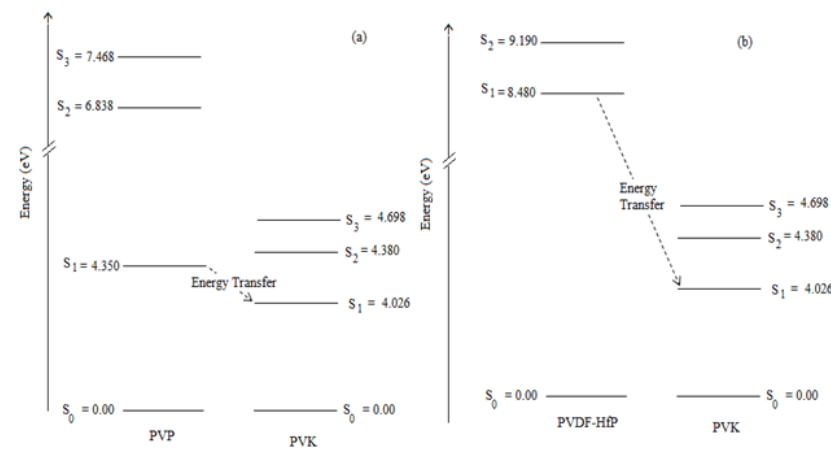

Fig. 3. Singlet-singlet energy level of photophysical process for (a) PVP $\rightarrow$ PVK and (b) PVDF-HfP $\rightarrow$ PVK energy transfer.

Secondly, the changes in physical bonding occur and increase the aggregation of luminescent chromophores. When PVK is blended with other polymers, such as PVDF-HfP and PVP, the changes occur in the excitation and emission spectra because of complex formation in the polymer blend system [17]. The recombination does not occur in the whole polymer solid. However, this recombination has occurred in certain regions which are known as chromophores. In organic polymer, the recombination centers strongly depend on the physical bonding or electron pairs. FTIR spectra have revealed the existence of some changes in the main vibrational absorption of the carbazole functional group. PVK:PVDF-HfP has the lowest main vibrational absorption intensity of the carbazole functional group, compared with PVK and PVK:PVP. Meanwhile, PVK:PVP shows an increase in the frequency of the vibrational group. Thus, these results confirm the formation of complexes in the polymer blend system. Changes in the physical bonding, as indicated in the FTIR spectrum, lead to the formation of new dopant emission centers in the polymer materials. The highest emission intensity of PVK:PVP may be attributed to the complex formation between the carbonyl group of PVP and the carbazole group of PVK, in which this complex formation is much higher compared with PVDF-HfP.

Thirdly, the states from the gap of the polymer blend systems change, and the existence of localized states in the disordered system consequently results in new optical absorption and luminescence spectra of the polymer 
blend systems [18]. In the polymer blend systems, the mixture of two polymers induces disorder in thin film solids. This induced disorder embarks on the band tail and extends into forbidden states known as Anderson localization states. This localized state in the band tail allows transition with delocalized states.

\section{Conclusions}

We have analysed the excitation and emission spectra of PVK, PVK:PVDF-HfP and PVK:PVP polymer blends. The result shows a discrepancy in the maximum excitation and emission for each samples. PVK:PVDFHfP has the highest excitation intensity. Based on semiempirical calculation, PVDF-HfP has shown the highest singlet energy which requires more energy to excite a donor from ground state to the excited state. However, in the emission spectrum, PVK:PVP has the highest emission intensity, followed by PVK:PVDF-HfP and PVK. These changes are due to the complex formation between polymer blends that create new aggregations of chromophores (shown in FTIR spectra). Furthermore, the existence of localized states in the disordered system consequently results in new optical absorption and luminescence spectra.

\section{Acknowledgments}

This work was funded by The Fundamental Grant Scheme (FRGS): 600-RMI/ST/FRGS $5 / 3 /$ Fst(198/2010). The authors would like to thank Faculty of Applied Sciences, UiTM, SIRIM AMREC Kulim and NanoScience Tech, Institutes of Science (IOS) for giving permission to carry out some measurements.

\section{References}

[1] A.N. Alias, M.K. Harun, M. Yahya, Z.M. Zabidi, T.I.T. Kudin, Advanced Materials Research 501, 39 (2012).
[2] S.-J. Yoon, H. Chun, M.-S. Lee, N. Kim, Synthetic Metals 159, 518 (2009).

[3] Z.M. Zabidi, A.N. Alias, S.H. Khalid, N.F.M. Sahapini, AIP Conference Proceedings 1482, 667 (2012).

[4] M.M. Mbarek, F. Massuyeau, J.-L. Duvail, J. Wery, E. Faulques, K. Alimi, Journal of Applied Polymer Science 130, 2839 (2013).

[5] A. Alias, Z. Zabidi, A. Ali, M. Harun, M. Yahya, International Journal of Applied Science and Technology 3, 11 (2013).

[6] J. Ridley, M. Zerner, Theoretica chimica acta 32, 111 (1973).

[7] R.A. Street, Advances in Physics 25, 397 (1976).

[8] R.A. Street, Phys. Rev. B 17, 3984 (1978).

[9] L. Bakueva, D. Matheson, S. Musikhin, E.H. Sargent, Synthetic Metals 126, 207 (2002).

[10] N. Ballav, M. Biswas, Synthetic Metals 132, 213 (2003).

[11] M. Baibarac, I. Baltog, S. Lefrant, P. Gomez-Romero, Polymer 48, 5279 (2007).

[12] D. Saikia, A. Kumar, Electrochimica Acta 49, 2581 (2004).

[13] A.N. Alias, M. Harun, T. Kudin, Z. Zabidi, A.M.M. Ali, M.Z.A. Yahya, Advanced Materials Research 652, 550 (2013).

[14] C.-J. Chang, Y.-Y. Cheng, M.H. Wang, C.S. Tuan, Thin Solid Films 477, 14 (2005).

[15] R.P. Haugland, J. Yguerabide, L. Stryer, Proceedings of the National Academy of Sciences 63, 23 (1969).

[16] V.Y. Artyukhov, G.V. Mayer, Russian Physics Journal 55, 835 (2012)

[17] I.S. Elashmawi, N.A. Hakeem, Polymer Engineering and Science 48, 895 (2008).

[18] A.N. Alias, M. Yahya, T. Kudin, Z. Zabidi, M. Harun, Advanced Materials Research 488, 628 (2012). 